\title{
EFeito de Herbicidas sobre a Comunidade de ARTRópodes do Solo do Feijoeiro Cultivado em Sistema de Plantio Direto e Convencional ${ }^{1}$
}

\author{
Effect of Herbicides on Soil Arthropod Community of Bean Cultivated under No-Tillage and \\ Conventional Systems
}

\author{
PEREIRA, J.L. ${ }^{2}$, PICANÇO, M.C. ${ }^{3}$, SILVA, A.A. ${ }^{4}$, BARROS, E.C. ${ }^{2}$, XAVIER, V.M. ${ }^{5}$ e \\ GONTIJO, P.C. ${ }^{5}$
}

\begin{abstract}
RESUMO - Neste trabalho, objetivou-se avaliar os efeitos da mistura dos herbicidas fomesafen + fluazifop sobre a comunidade de artrópodes do solo cultivado com feijão nos sistemas plantio direto e convencional. O experimento foi realizado em Argissolo Vermelho-Amarelo distrófico de boa fertilidade, em Coimbra, MG. Duas semanas após o plantio foi aplicada a mistura comercial dos herbicidas fluazifop + fomesafen, denominada Robust ${ }^{\circledR}$, na dose de $0,8 \mathrm{~L} \mathrm{ha}^{-1}$; em cada sistema de cultivo foi mantida uma testemunha sem aplicação de herbicida. O delineamen to experimental foi o de blocos casualizados, com cinco repetições. As populações de artrópodes do solo foram amostradas aos 1, 8, 21 e 42 dias após a aplicação do herbicida. Os dados foram submetidos à análise de variáveis canônicas (CVA), que é uma técnica de ordenação indireta que reduz a dimensionalidade do conjunto dos dados originais em um conjunto de variáveis que podem ser usadas para ilustrar graficamente as posições relativas e as orientações das médias das respostas da comunidade em cada tratamento sob comparação. As diferenças entre os tratamentos na abundância dos artrópodes selecionados foram determinadas pela análise de variância por medida repetida, considerando a data de amostragem como a medida repetida. A análise das variáveis canônicas CVA para os sistemas de plantio e aplicação de herbicida indicou diferenças na densidade de artrópodes coletados nos diferentes tratamentos estudados, considerando a composição e a abundância das espécies. Foram observadas, por meio do diagrama de ordenação, diferenças no conjunto de artrópodes do solo coletados nos dois sistemas de plantio. A aplicação dos herbicidas afetou a densidade dos artrópodes associados ao feijoeiro em ambos os sistemas de cultivo, com exceção do Solenopsis sp. O sistema de plantio afetou a densidade de todas as espécies estudadas.
\end{abstract}

Palavras-chave: fluazifop-p-butyl, Fomesafen, Phaseolus vulgaris.

\begin{abstract}
The aim of this research was to evaluate the effect of herbicide mixture (fomesafen + fluazifop) on the soil arthropod communities associated to the common bean in two cropping systems (conventional and no-tillage). The experiment was carried out on Red-yellow Podzol, in Coimbra, Minas Gerais, Brazil. The studied treatments in both cropping system were represented by the mixture of herbicide Robust ${ }^{\circledR}$ (fluazifop + fomesafen) (dosage of $0.8 \mathrm{~L} \mathrm{ha}^{-1}$ ) applied two weeks after planting and control without herbicide application. A complete randomized block design with five replications was used in the experiment. The soil arthropod communities were sampled 1, 8, 21 and 42 days after herbicide application. The datawere submitted to the canonical variate analysis (CVA). CVA is an indirect ordination technique that reduces the size of the original data set. The CVA techniques can be used to illustrate graphically the relative positions and the coordinates of the community's structure for each treatment. The differences between
\end{abstract}

Recebido para publicação em 9.6.2006 e na forma revisada em 27.2.2007.

Estudante de Doutorado, Departamento de Fitotecnia, Universidade Federal de Viçosa - DFT/UFV, 36570-000 Viçosa-MG, <jardelmail@yahoo.com.br>, <ecristi@yahoo.com.br>; ${ }^{3}$ Professor Adjunto, Departamento de Biologia Animal - DBA/UFV, <picanço@ufv.br>; ${ }^{4}$ Professor Associado - DFT/UFV, <aasilva@ufv.br>; ${ }^{5}$ Estudante de Iniciação Científica - DBA/UFV, <vaniamxavier@yahoo.com.br>, <pablocgontijo@yahoo.com.br>. 


\begin{abstract}
the treatments in the abundance of the selected arthropods had been determined by repeated measure analysis of variance considering the sampling data as repeated measure. The canonical variate analysis for cropping systems and herbicide application indicated differences between the arthropod densities in the different treatments considering the composition and the species abundance. In this study, differences in the set data of collected soil arthropods were observed through ordination diagrams under the two cropping systems. Herbicide application affected all arthropods associated to the common beans in both cropping systems, except Solenopsis $s p$. The cultivation system affected the densities of all species studied.
\end{abstract}

Keywords: fluazifop-p-butyl, Fomesafen, Phaseolus vulgaris.

\section{INTRODUÇÃO}

A cultura do feijão (Phaseolus vulgaris) é de expressiva importância econômica no cenário nacional, destacando-se tanto pelo seu grande uso alimentar quanto pela extensão da área cultivada (Rapassi et al., 2003). Segundo dados da Companhia Nacional de Abastecimento (CONAB), a produtividade média do feijoeiro da primeira safra de 2005/06 foi de $914 \mathrm{~kg}$ ha, o que representa, em média, menos de um terço do potencial de rendimento dos últimos cultivares lançados. Dentre os fatores que contribuem para a baixa produtividade do feijoeiro no Brasil, destaca-se a utilização de forma incorreta dos pesticidas, que resulta em controle ineficiente de pragas, doenças e plantas daninhas, além do alto risco de poluição ambiental. Esses produtos devem ter seu uso fundamentado no tocante à seletividade e eficiência no controle do alvo biológico, com o mínimo de efeito sobre inimigos naturais, decompositores e demais agentes benéficos presentes na área de aplicação (Cruz, 1986).

Alterações na cadeia alimentar de artrópodes do solo, na composição e na diversidade de espécies associadas às culturas foram relatadas por diversas pesquisas em conseqüência de práticas agrícolas utilizadas (Giller, 1997; Pereira et al., 2004; Robertson, 1994). Considerando os pesticidas, Belden \& Lydy (2000) afirmam que o uso de herbicidas pode exercer efeitos nocivos sobre a entomofauna, embora a magnitude de respostas possa estar mais diretamente ligada a efeitos indiretos decorrentes de mudanças no habitat. Alguns desses efeitos podem ser devido à perda da cobertura vegetal, exercida pelas plantas daninhas, e pela eliminação da fonte de alimentos de alguns artrópodes, podendo esses efeitos ser mais significativos que os efeitos diretos resultantes da sua composição química. Dentre os herbicidas recomendados para a cultura do feijão, destaca-se a mistura comercial de fomesafen com o fluazifop-p-butyl (Robust ${ }^{\circledR}$ ), que permite o controle simultâneo de plantas daninhas dicotiledôneas e gramíneas (Silva et al., 2003).

Com relação aos sistemas de cultivo, diversos estudos têm demonstrado que as densidades populacionais de pragas e de inimigos naturais se diferem nos sistemas plantio direto e convencional (Risch et al., 1983; Andow, 1991; Cividanes \& Barbosa, 2001). Nesta pesquisa, objetivou-se avaliar o impacto da mistura comercial dos herbicidas fluazifop-pbutyl + fomesafen, denominada Robust ${ }^{\circledR}$, sobre a comunidade de artrópodes do solo cultivado com a cultura do feijão nos sistemas de plantio direto e convencional.

\section{MATERIAL E MÉTODOS}

O experimento foi realizado no período de agosto a dezembro de 2005 na Estação Experimental da Universidade Federal de Viçosa, localizada no municipio de Coimbra-MG $\left(20^{\circ} 45^{\prime}\right.$ de latitude sul, 42 ${ }^{\circ} 51^{\prime}$ de longitude oeste e $651 \mathrm{~m}$ de altitude). O solo da área foi classificado como Argissolo Vermelho-Amarelo distrófico textura argilosa, fase terraço. A caracterização química e textural do solo nos dois sistemas de cultivo se encontra na Tabela 1.

Para preparo do solo no plantio direto, foi realizada a dessecação química com os herbicidas glyphosate $+2,4-\mathrm{D}\left(1,44+0,335 \mathrm{~kg} \mathrm{ha}^{-1}\right.$, respectivamente) em mistura no tanque, três semanas antes da semeadura. No plantio convencional, utilizou-se o preparo mecânico do solo com uma aração e duas gradagens, sete dias antes da semeadura. Para controle 
Tabela 1 - Composição química e textural da camada de 0-10 $\mathrm{cm}$ de profundidade do solo argiloso proveniente dos sistemas plantio direto (SPD) e convencional (SPC) utilizados no experimento (Coimbra-MG, 2005)

\begin{tabular}{|c|c|c|c|c|c|c|c|c|c|c|}
\hline \multicolumn{11}{|c|}{ Sistema de Plantio Dire to } \\
\hline \multicolumn{11}{|c|}{ Fração Mineral (\%) } \\
\hline \multicolumn{2}{|c|}{ Areia fina } & \multicolumn{3}{|c|}{ Areia grossa } & \multicolumn{3}{|c|}{ Silte } & \multicolumn{2}{|c|}{ Argila } & Textura \\
\hline \multicolumn{2}{|c|}{10} & \multicolumn{3}{|c|}{16} & \multicolumn{3}{|c|}{19} & \multicolumn{2}{|c|}{55} & Argilosa \\
\hline \multicolumn{11}{|c|}{ Análise Química } \\
\hline $\mathrm{pH}$ & $\mathrm{P}$ & $\mathrm{K}^{+}$ & $\mathrm{H}+\mathrm{Al}$ & $\mathrm{Al}^{3+}$ & $\mathrm{Ca}^{2+}$ & $\mathrm{Mg}^{2+}$ & $\mathrm{CTC} \mathrm{Tt}$ & $\mathrm{V}$ & $\mathrm{M}$ & $\mathrm{MO}$ \\
\hline$\left(\mathrm{H}_{2} \mathrm{O}\right)$ & \multicolumn{2}{|c|}{$\left(\mathrm{mg} \mathrm{dm}^{3}\right)$} & \multicolumn{5}{|c|}{$\left(\mathrm{cmol}_{\mathrm{c}} \mathrm{dm}^{-3}\right)$} & \multicolumn{2}{|c|}{$(\%)$} & $\left(\right.$ dag kg $\left.^{-1}\right)$ \\
\hline 5,1 & 14,4 & 54 & 4,46 & 0,3 & 1,6 & 0,6 & 6,80 & 34 & 11 & 2,94 \\
\hline \multicolumn{11}{|c|}{ Sistema de Plantio Convencional } \\
\hline \multicolumn{11}{|c|}{ Fração Mineral (\%) } \\
\hline \multicolumn{2}{|c|}{ Areia fina } & \multicolumn{3}{|c|}{ Areia grossa } & \multicolumn{3}{|c|}{ Silte } & \multicolumn{2}{|c|}{ Argila } & Textura \\
\hline \multicolumn{2}{|c|}{15} & \multicolumn{3}{|c|}{29} & \multicolumn{3}{|c|}{11} & \multicolumn{2}{|c|}{45} & Argilosa \\
\hline \multicolumn{11}{|c|}{ Análise Química } \\
\hline $\mathrm{pH}$ & $\mathrm{P}$ & $\mathrm{K}^{+}$ & $\mathrm{H}+\mathrm{Al}$ & $\mathrm{Al}^{3+}$ & $\mathrm{Ca}^{2+}$ & $\mathrm{Mg}^{2+}$ & CTC Tt & $\mathrm{V}$ & $\mathrm{M}$ & $\mathrm{MO}$ \\
\hline$\left(\mathrm{H}_{2} \mathrm{O}\right)$ & \multicolumn{2}{|c|}{$\left(\mathrm{mg} \mathrm{dm}^{3}\right)$} & \multicolumn{5}{|c|}{$\left(\mathrm{cmol}_{\mathrm{c}} \mathrm{dm}^{-3}\right)$} & \multicolumn{2}{|c|}{$(\%)$} & $\left(\right.$ dag $\left.\mathrm{kg}^{-1}\right)$ \\
\hline 5,3 & 8,3 & 68 & 4,62 & 0,2 & 2,0 & 0,7 & 7,49 & 38 & 7 & 2,62 \\
\hline
\end{tabular}

* Análises realizadas nos Laboratórios de Análises Físicas e Químicas de Solo do Departamento de Solos da UFV, segundo a metodologia descrita pela Empresa Brasileira de Pesquisa Agropecuária-Embrapa (1997).

das plantas daninhas na cultura do feijão, em ambos os sistemas de plantio foi utilizada a mistura comercial dos herbicidas fluazifop-pbutyl + fomesafen $\left(200+250 \mathrm{~g} \mathrm{~L}^{-1}\right)$, na dosagem comercial de $0,8 \mathrm{~L} \mathrm{ha}^{-1}$. Em cada sistema de cultivo foi mantida uma testemunha sem aplicação de herbicida. O delineamento experimental foi o de blocos casualizados, com cinco repetições. A aplicação dos herbicidas foi realizada aos 14 dias após a emergência (DAE) do feijoeiro, em ambos os sistemas de cultivo. $\mathrm{Na}$ aplicação dos herbicidas foi utilizado pulverizador costal pressurizado com $\mathrm{CO}_{2}$, mantido à pressão constante de $200 \mathrm{kPa}$, equipado com dois bicos TT 110.02 , espaçados de $1,0 \mathrm{~m}$ e calibrados para aplicar o equivalente a $100 \mathrm{~L} \mathrm{ha}^{-1}$ de calda. As condições no momento da aplicação foram de céu claro, solo úmido, velocidade do vento inferior a $5 \mathrm{~km} \mathrm{~h}^{-1}$, temperatura do ar de $24,8^{\circ} \mathrm{C}$ e umidade relativa de $74 \%$. Os dados de temperatura e precipitação diária referentes ao período de condução do experimento estão representados na Figura 1.

As populações de artrópodes do solo foram amostradas aos 1, 8, 21 e 42 dias após a aplicação do herbicida, sendo as amostras referentes à comunidade de artrópodes do solo coletadas por meio da retirada de blocos de solo com aproximadamente $30 \mathrm{~cm}$ de profundidade e $10 \mathrm{~cm}$ de diâmetro. Esse material foi levado ao Laboratório de Manejo Integrado de Pragas, pertencente à UFV, onde foram submetidos ao funil de berleze, conforme proposto

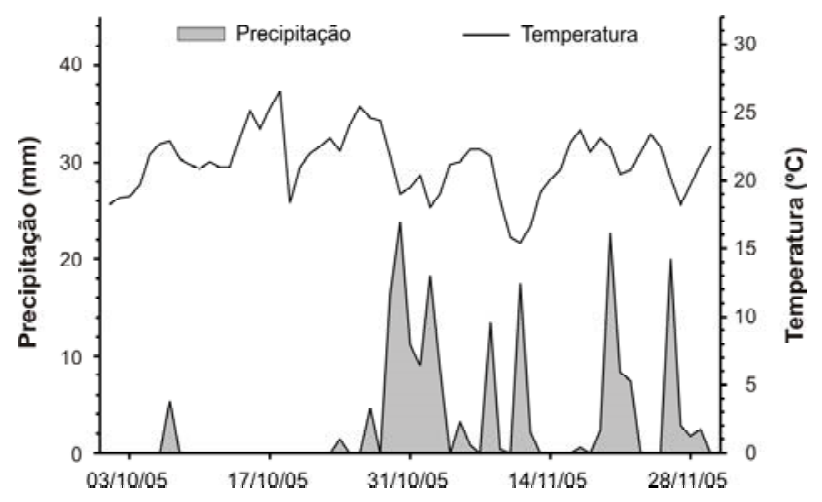

Figura 1 - Precipitação e temperatura média observadas durante a condução do experimento (Coimbra-MG, 2005).

Planta Daninha, Viçosa-MG, v. 25, n. 1, p. 61-69, 2007 
por Wardle et al. (1993). As amostras provenientes da coleta no campo por berleze foram conservadas em potes de vidro com álcool $70 \%$, sendo posteriormente transferidas para placas de Petri e submetidas à contagem do número total de artrópodes, utilizando-se o microscópio estereoscópio com aumento fixado de $12 \mathrm{X}$. Após isso, os artrópodes coletados foram então encaminhados para taxonomistas, para identificação.

O impacto dos sistemas de cultivo e da aplicação do herbicida sobre as comunidades de artrópodes do solo foi determinado pela comparação da abundância relativa de cada espécie entre os tratamentos. Os dados foram inicialmente submetidos a um processo seletivo que determina quais espécies que mais explicam a variância observada (PROC STEPDISC com seleção STEPWISE; SAS, 2001). As espécies foram selecionadas de acordo com dois critérios coincidentes no presente caso: 1) o nivel de significância do teste $\mathrm{F}$ da análise de covariância, em que as espécies escolhidas agem como covariáveis e os tratamentos como variáveis dependentes; e 2) a correlação quadrada parcial, predizendo os efeitos dos tratamentos a partir das espécies, controlada pelo efeito causado pelas espécies já selecionadas pelo modelo (SAS, 2001).

Os dados das espécies selecionadas foram submetidos à análise de variáveis canônicas (CVA), que é uma técnica de ordenação indireta que reduz a dimensionalidade do conjunto dos dados originais em um conjunto de variáveis que podem ser usadas para ilustrar graficamente as posições relativas e as orientações das médias das respostas da comunidade em cada tratamento sob comparação (Kedwards et al., 1999). A significância da diferença (indicada pela ordenação) entre grupos devido ao tratamento foi determinada pela comparação dois a dois dos tratamentos pelo teste $\mathrm{F}$ aproximado $(\mathrm{p}<0,05)$, usando a distância de Mahalanobis entre as respectivas classes de médias canônicas. As análises foram feitas usando o procedimento CANDISC do pacote estatístico do SAS (SAS, 2001).

Os dados das principais espécies que levaram ao aparecimento de diferenças entre os tratamentos foram individualmente submetidos à análise de variância por medidas repetidas, para ver por quanto tempo perdura o distúrbio e uma eventual recuperação das espécies ao estresse. Uma vez que a amostragem dos artrópodes foi realizada no mesmo campo várias vezes, a análise de variância por medida repetida é recomendada para evitar o problema de pseudo-replicação no tempo (Hurlbert, 1984; Stewart-Oater et al., 1986; Green, 1993; Paine, 1996). Essas análises foram feitas usando o procedimento ANOVA do SAS com a especificação PROFILE, como sugerido por von Ende (1993). A normalidade e a homogeneidade das variâncias foram testadas usando o procedimento UNIVARIATE (SAS, 2001).

\section{RESULTADOS E DISCUSSÃO}

No total, 127 espécies de artrópodes foram coletadas nos diferentes tratamentos. Desse conjunto, apenas entraram na análise aquelas espécies cuja freqüência de coleta tenha sido maior que $10 \%$ em pelo menos um dos tratamentos (Tabela 2). Dessas espécies, Hypoaspis sp., Galumnidae, Parasitidae, Hypogastrura sp., Solenopsis sp., Larva de Crisomelidae, Larva de Stafilinidae e Entomobryidae foram as que melhor explicaram a variação observada nos quatro tratamentos, sendo selecionadas para análise adicional (Tabela 3 ).

A análise das variáveis canônicas CVA para sistema de plantio (convencional e direto) e aplicação de herbicida (com ou sem aplicação de Robust ${ }^{\circledR}$ ) indicou diferenças significativas entre os tratamentos, considerando a composição e a abundância das espécies (Wilks' lambda $=0,0354 ; \mathrm{F}=18,10 ; \mathrm{gl}(\mathrm{num} / \mathrm{den})=$ 24/200, 7; P < 0,0001). Dois eixos canônicos foram calculados, sendo ambos significativos $(\mathrm{P}<0,0001$ e $\mathrm{P}=0,0163$ ) (Tabela 4).

Com base no coeficiente canônico (entre estrutura canônica), as espécies que mais contribuíram para a divergência entre os tratamentos no eixo 1 foram Larva de Stafilinidae, Galumnidae, Entomobryidae e Larva de Crisomelidae. Parasitidae contribuiu negativamente na explicação do conjunto dos dados no eixo 1. No eixo 2, Parasitidae, Hypogastrura sp. e Solenopsis sp. contribuíram para a divergência entre os tratamentos, 
enquanto Entomobryidae, Galumnidae, Larva de Crisomelidae, Larva de Stafilinidae e Isotoma sp. contribuíram negativamente na explicação do conjunto dos dados (Tabela 4). O diagrama de ordenação derivado da análise das variáveis canônicas foi feito com os dois eixos canônicos significativos, que juntos explicam 99,3\% do total da variância avaliada. O diagrama mostrou diferenças significativas no conjunto de artrópodes encontrados nos diferentes sistemas de cultivo. Segundo MacLaughlin \& Mineau (1995) e Andersen (1999), invertebrados do solo são afetados pelo sistema de plantio utilizado. A aplicação dos herbicidas afetou o conjunto de artrópodes associados ao feijoeiro em ambos os sistemas de cultivo (Figura 2). Alguns trabalhos têm evidenciado propriedades inseticidas para alguns herbicidas, como o de Soares et al. (1995), que observaram redução na população dos predadores C. sanguinea e $D$. lineare após a aplicação dos herbicidas MSMA e fluazyfop-p-butyl, e Pereira et al. (2004), que constataram redução nas populações de ácaros e formigas do solo após a aplicação da mistura dos herbicidas nicosulfuron + atrazine.

Tabela 2 - Abundância e freqüência dos artrópodes mais abundantes coletados no solo do feijoeiro cultivado nos sistemas de plantio convencional e direto (Coimbra-MG, 2005)

\begin{tabular}{|c|c|c|c|c|c|}
\hline \multirow{3}{*}{ Artrópode } & \multicolumn{4}{|c|}{$\begin{array}{c}\text { Abundância } \\
\text { (média } \pm \text { erro-padrão da média do número de indivíduos/amostra) }\end{array}$} & \multirow{3}{*}{ Freq.* } \\
\hline & \multicolumn{2}{|c|}{ Plantio Convencional } & \multicolumn{2}{|c|}{ Plantio Direto } & \\
\hline & Com herbicida & Sem herbicida & Com herbicida & Sem herbicida & \\
\hline Cheyletus sp. (Acari: Cheyletidae) & $12,05 \pm 1,69$ & $28,85 \pm 4,21$ & $19,55 \pm 2,00$ & $37,30 \pm 2,28$ & 100,00 \\
\hline Hypoaspis sp. (Acari:Laelapidae) & $1,65 \pm 0,21$ & $4,60 \pm 0,73$ & $2,35 \pm 0,47$ & $8,40 \pm 0,78$ & 91,25 \\
\hline Galumnidae (Acari: Oribatida) & $0,85 \pm 0,21$ & $3,90 \pm 0,49$ & $0,80 \pm 0,16$ & $8,05 \pm 0,84$ & 80,00 \\
\hline Parasitidae (Acari) & $6,15 \pm 1,18$ & $25,00 \pm 4,93$ & $19,50 \pm 1,78$ & $33,85 \pm 1,67$ & 97,50 \\
\hline Larva de Bostrichidae (Coleóptera) & $0,10 \pm 0,07$ & $0,80 \pm 0,20$ & $0,05 \pm 0,05$ & $0,75 \pm 0,25$ & 27,50 \\
\hline Hypogastrura sp. (Collembola: Hypogastruridae) & $4,50 \pm 0,79$ & $15,65 \pm 2,76$ & $9,15 \pm 1,43$ & $33,10 \pm 2,73$ & 97,50 \\
\hline Entomobryidae (Collembola) & $0,40 \pm 0,15$ & $2,30 \pm 0,44$ & $0,05 \pm 0,05$ & $3,75 \pm 0,58$ & 46,25 \\
\hline Orchesella sp. (Collembola: Orchesellinae) & $1,60 \pm 0,33$ & $4,30 \pm 0,58$ & $2,05 \pm 0,52$ & $6,40 \pm 0,87$ & 83,75 \\
\hline Solenopsis sp. (Formicidae: Myrmiciinae) & $3,75 \pm 1,07$ & $4,35 \pm 2,12$ & $11,10 \pm 2,73$ & $11,35 \pm 3,02$ & 90,00 \\
\hline Neivamyrmex sp. (Hymenoptera: Formicidae) & $0,10 \pm 0,07$ & $1,25 \pm 0,55$ & $0,90 \pm 0,40$ & $0,35 \pm 0,07$ & 22,50 \\
\hline Larva de Crisomelidae (Coleoptera) & $0,70 \pm 0,22$ & $4,85 \pm 0,42$ & $1,05 \pm 0,25$ & $8,30 \pm 0,84$ & 78,75 \\
\hline Larva de Syrphidae (Diptera) & $0,10 \pm 0,07$ & $1,95 \pm 0,38$ & $0,40 \pm 0,17$ & $3,20 \pm 0,58$ & 50,00 \\
\hline Larva de Scarabaeidae (Coleoptera) & $0,10 \pm 0,10$ & $0,20 \pm 0,16$ & $0,40 \pm 0,30$ & $1,20 \pm 0,72$ & 13,75 \\
\hline Larva de Stafilinidae (Coleoptera) & $0,55 \pm 0,21$ & $3,30 \pm 0,52$ & $0,50 \pm 0,17$ & $5,80 \pm 0,72$ & 65,00 \\
\hline Entomobryidae (Collembola) & $0,10 \pm 0,07$ & $1,55 \pm 0,44$ & $0,10 \pm 0,07$ & $1,85 \pm 0,32$ & 75,00 \\
\hline
\end{tabular}

* Freqüência $(\%)=$ (número de amostras com a espécie/número total de amostras ) x 100 .

Tabela 3 - Espécies de artrópodes selecionadas pelo STEPWISE com procedimento STEPDISC do SAS a serem incluídas na análise de variáveis canônicas, obtendo-se a máxima discriminação entre os tratamentos (Coimbra-MG, 2005)

\begin{tabular}{|c|c|c|c|c|c|c|c|}
\hline \multirow{2}{*}{ Passo } & \multicolumn{2}{|l|}{ Variável } & \multirow[b]{2}{*}{$\mathrm{R}^{2}$ parcial } & \multicolumn{2}{|c|}{$\begin{array}{l}\text { Test } \mathrm{F}-\mathrm{da} \text { análise de } \\
\text { covariância }\end{array}$} & \multicolumn{2}{|c|}{ Correlação quadrada parcial } \\
\hline & Adicionada & Retirada & & $\mathrm{F}$ & $P$ & $\begin{array}{l}\text { Média da correlação } \\
\text { canônica quadrada }\end{array}$ & $p$ \\
\hline 1 & Larva de Crisomelidae & - & 0,6732 & 52,17 & $<0,0001$ & 0,224 & $<0,0001$ \\
\hline 2 & Galumnidae & - & 0,4256 & 18,52 & $<0,0001$ & 0,274 & $<0,0001$ \\
\hline 3 & Lavva de Stafilinidae & - & 0,5959 & 36,37 & $<0,0001$ & 0,312 & $<0,0001$ \\
\hline 4 & Entomobryidae & - & 0,1638 & 4,77 & 0,0043 & 0,322 & $<0,0001$ \\
\hline 5 & Isotoma sp. & - & 0,1929 & 5,73 & 0,0014 & 0,336 & $<0,0001$ \\
\hline 6 & Parasitidae & - & 0,1593 & 4,48 & 0,0061 & 0,385 & $<0,0001$ \\
\hline 7 & Hypogastrura sp. & - & 0,1027 & 2,67 & 0,0542 & 0,408 & $<0,0001$ \\
\hline 8 & Solenopsis sp. & - & 0,0838 & 2,10 & 0,1077 & 0,431 & $<0,0001$ \\
\hline
\end{tabular}


Tabela 4 - Eixos canônicos e seus coeficientes (entre estrutura canônica) relativos aos efeitos dos herbicidas em artrópodes do solo coletados em dois sistemas de plantio: convencional e plantio direto (Coimbra-MG, 2005)

\begin{tabular}{|l|r|r|}
\hline \multirow{2}{*}{\multicolumn{1}{c|}{$\begin{array}{c}\text { Variável } \\
\text { (espécie de artrópode) }\end{array}$}} & \multicolumn{2}{c|}{ Eixo canônico } \\
\cline { 2 - 3 } & 1 & \multicolumn{1}{c|}{2} \\
\hline Hypoaspis sp. & 0,180 & $-0,082$ \\
\hline Galumnidae & 0,444 & $-0,060$ \\
\hline Parasitidae & $-0,008$ & 0,065 \\
\hline Hypogastrura sp. & 0,021 & 0,054 \\
\hline Solenopsis sp. & 0,005 & 0,059 \\
\hline Larva de Crisomelidae & 0,283 & $-0,108$ \\
\hline Larva de Stafilinidae & 0,444 & $-0,264$ \\
\hline Entomobryidae & 0,334 & $-0,010$ \\
\hline F & 17,21 & 2,09 \\
\hline gl (numerador; denominador) & $24 / 189,1$ & $14 / 132$ \\
\hline P & $<0,0001$ & 0,0163 \\
\hline Correlação canônica parcial & 0,976 & 0,993 \\
\hline
\end{tabular}

* Significância a 5\% pelo teste $\mathrm{F}$.

A análise de medida repetida mostrou que os sistemas de plantio afetaram todas as espécies estudadas: Hypoaspis sp. ( $\mathrm{F}=31,67$; $\mathrm{P}=0,0014)$, Galumnidae $(\mathrm{F}=11,16 ; \mathrm{P}=0,01)$, Parasitidae $(\mathrm{F}=22,16 ; \mathrm{P}=0,0022)$, Hypogastrura sp. $(\mathrm{F}=12,10 ; \mathrm{P}=0,01)$, Solenopsis sp. $(\mathrm{F}=17,16 ; \mathrm{P}=0,02)$, Larva de Crisomelidae $(F=13,00 ; P=0,02)$, Larva de Stafilinidae $(\mathrm{F}=7,22 ; \mathrm{P}<0,001) \mathrm{e}$ Entomobryidae $(\mathrm{F}=10,00 ; \mathrm{P}=0,02)$. Também, os herbicidas, com exceção de Solenopsis sp., afetaram todas as es pécies estudadas: Hypoaspis sp. $(\mathrm{F}=21,67 ; \mathrm{P}<0,001)$, Galumnidae $(\mathrm{F}=13,11 ; \mathrm{P}=0,02)$, Parasitidae $(\mathrm{F}=12,14 ; \mathrm{P}=0,017)$, Hypogastrura sp. $(\mathrm{F}=7,21 ; \mathrm{P}=0,002)$, Larva de Crisomelidae $(F=8,70 ; P=0,02)$, Larva de Stafilinidae $(F=11,33 ; P=0,03)$ e Entomobryidae $(F=3,14$; $\mathrm{P}=0,0007)$. As datas de amostragens afetaram Hypoaspis sp. $(\mathrm{F}=4,25 ; \mathrm{P}<0,001)$ e Solenopsis sp. $(\mathrm{F}=5,03 ; \mathrm{P}=0,03)$. Nas interações entre tratamentos $\mathrm{x}$ datas de amostragem, resultados significativos foram encontrados em Hypoaspis sp. ( $\mathrm{F}=1,47, \mathrm{P}<0,001$ e $\mathrm{F}=2,15$; $\mathrm{P}=0,008$, respectivamente) e Solenopsis $\mathrm{sp}$. $(\mathrm{F}=3,17, \mathrm{P}=0,03$ e $\mathrm{F}=1,02 ; \mathrm{P}=0,02$, respectivamente). A variação significativa da densidade média das espécies Hypoaspis sp. e Solenopsis sp. ao longo das datas de avaliação nos tratamentos estudados (Figura 3) pode estar correlacionada com os fatores climatológicos (Figura 1). Observa-se que essas espécies reduziram suas densidades médias com o aumento dos indices pluviométricos observados no final do experimento. Resultados semelhantes foram obtidos por Sanders \& Gordon (2004), que observaram reduções nas densidades de várias espécies de Formicidae em anos agrícolas com maiores volumes de precipitação. Segundo estes autores, esse fato pode estar correlacionado com o aumento do número de colônias inundadas, principalmente aquelas colônias mais novas, as quais são mais suscetiveis ao inundamento.

As espécies que mais contribuíram para a significância dos eixos canônicos apresentaram maior densidade média no sistema de plantio direto, comparado ao sistema de plantio convencional, em praticamente todas as datas avaliadas (Figura 3). Clark et al. (1997) mostraram que a densidade de carabídeos, formigas e aranhas aumenta com práticas agrícolas que reduzem a movimentação do solo. Esse fato pode estar relacionado com os fatores abióticos do solo, como o aumento da umidade, o incremento dos teores de matéria orgânica e a diminuição das temperaturas máximas do solo (Salton \& Mielniczuk, 1995), os quais contribuem para o incremento da riqueza e densidade de espécimes de artrópodes do solo (Phillips \& Cobb, 2005).

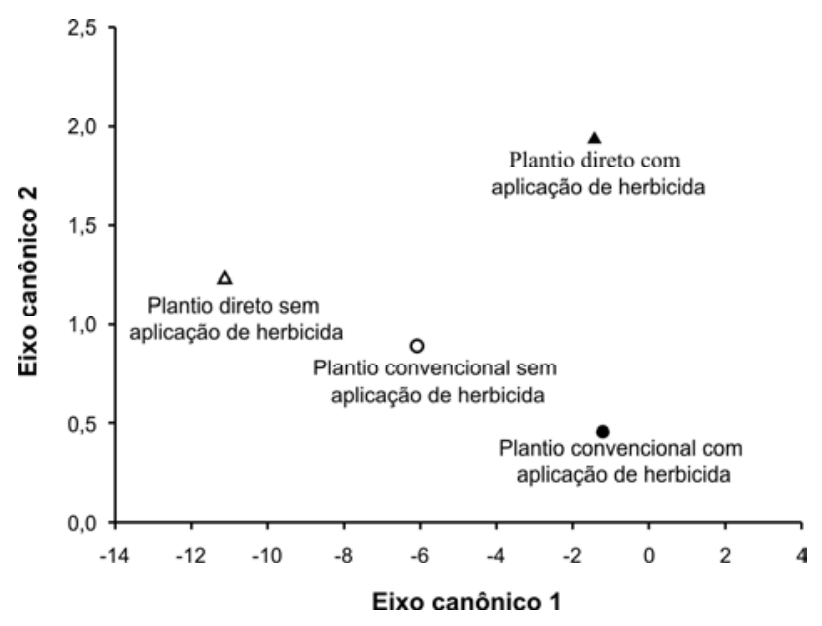

Figura 2 - Digrama de Ordenação mostrando a discriminação nas parcelas com e sem aplicação de herbicida nos sistemas de plantio convencional e direto. Todos os tratamentos diferiram entre si significativamente pelo teste $\mathrm{F}(\mathrm{P}<0,05)$, com base na distância de Mahalanobis entre as médias das classes (Coimbra-MG, 2005). 


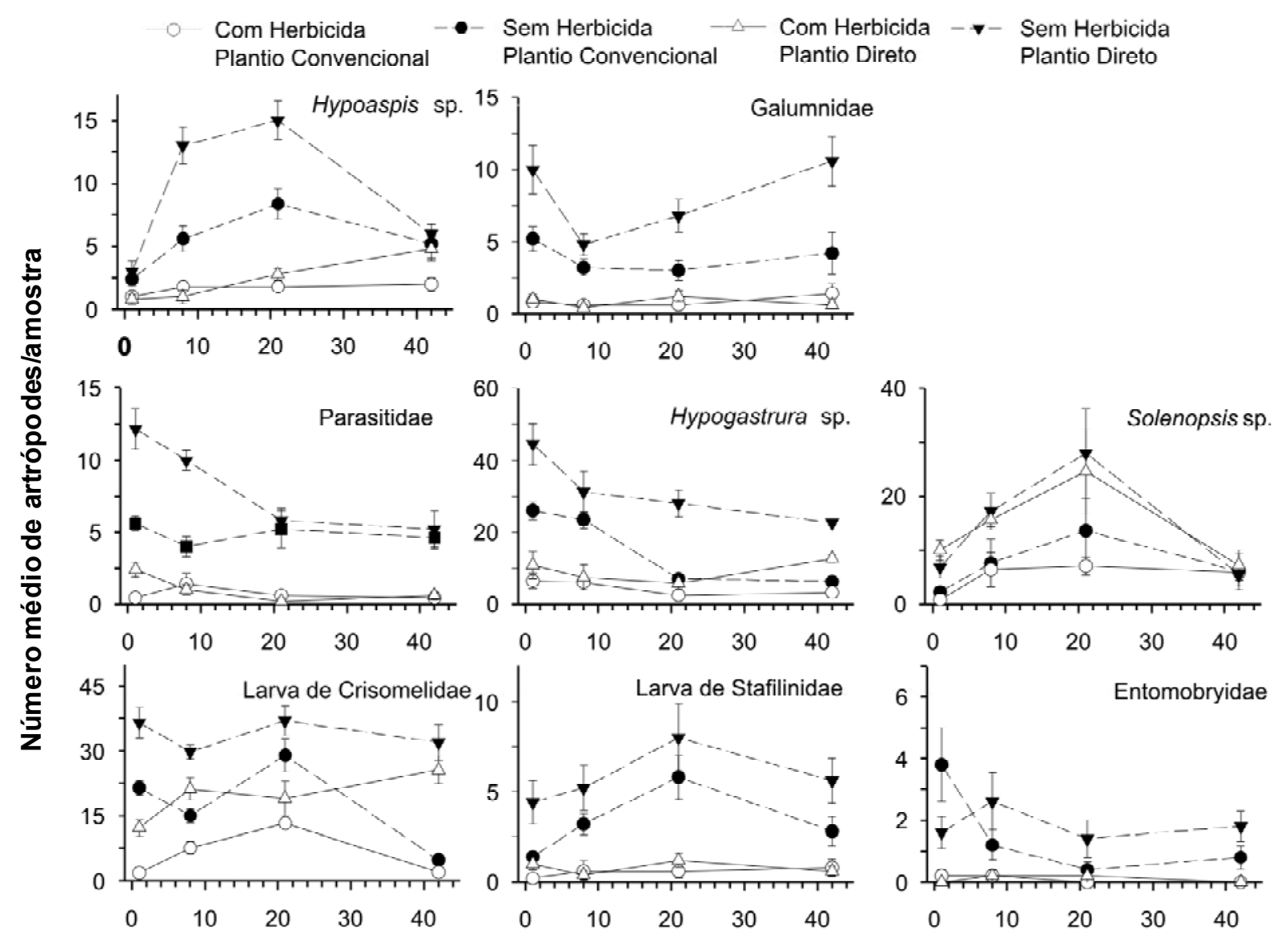

Dias após a aplicação

Figura 3 - Abundância das principais espécies de artrópodes do solo (média \pm EPM) coletadas na cultura do feijão em parcelas sob dois sistemas de plantio, com e sem aplicação de herbicidas (Coimbra-MG, 2005).

No sistema de cultivo convencional, o uso da mistura comercial dos herbicidas fluazifopp-butyl + fomesafen provocou redução na abundância de Hypoaspis sp., Galumnidae, Parasitidae e Larva de Stafilinidae até 42 dias depois da aplicação. As espécies Hypogastrura sp., Larva de Crisomelidae e Entomobryidae tiveram reduções iniciais, mas logo aos oito dias após a aplicação dos herbicidas as populações já retornaram à condição de equilíbrio (Figura 3). No sistema de plantio direto, a aplicação dos herbicidas contribuiu para a redução da abundância de Galumnidae, Parasitidae, Hypogastrura sp., Larva de Stafilinidae e Entomobryidae até os 42 dias após a aplicação, ao passo que a redução nas espécies Hypoaspi sp. e Larva de Crisomelidae foi observada até 21 dias após a aplicação. A espécie de formicidae Solenopsis sp. não foi influenciada pelos herbicidas em ambos os sistemas de cultivo (Figura 3).

Possiveis causas da agressividade dos herbicidas sobre as populações de artrópodes do solo podem estar relacionadas tanto à molécula do fluazifo p-p-butyl quan to à do herbicida fomesafen. O fluazifop-p-butyl é um potente inibidor da sintese de acetil coenzima A carboxilase (ACCase) (Thill, 2003), presente também no metabolismo de artrópodes. Já o fomesafen pode apresentar agressividade a artrópodes devido ao seu mecanismo de ação, o qual atua inibindo a enzima protoporfirinogênio oxidase (Protox), fazendo com que haja acúmulo de protoporfirina em células tratadas com este herbicida. A protoporfirina é produzida pela via biossintética do ferro, a qual é vital para a maioria dos animais (Walker et al., 2001). Entretanto, em grandes quantidades ela 
passa a interagir com o oxigênio para produção de formas reativas e, conseqüentemente, peroxidação dos lipídeos e morte celular (Weller, 2003).

Outro fator que pode ter acarretado a redução na freqüência de coleta dos artrópodes do solo, resultante da aplicação dos herbicidas nos dois sistemas de cultivo, pode estar relacionado com a redução da comunidade de plantas daninhas presentes em cada sistema, das quais muito desses artrópodes utilizam tanto para alimentação como para abrigo (Smith \& McSorley, 2000; Araújo et al., 2004). Segundo Strong et al. (1984), 45\% das espécies de artrópodes utilizam as plantas como fonte de alimentação, seja pela sucção direta da seiva no caso dos insetos fitófagos ou pela decomposição de restos vegetais realizada pelos artrópodes detritívoros. Muitos desses artrópodes se alimentam exclusivamente de plantas daninhas, podendo ter sua sobrevivência comprometida pelo controle dessas plantas invasoras através da aplicação de herbicidas, contribuindo assim para o desequilíbrio da entomofauna, ocasionando possiveis problemas com insetos-praga (Capinera, 2005).

A mortalidade observada até os 42 dias após a aplicação da mistura dos herbicidas, das espécies Hypoaspis sp., Galumnidae, Parasitidae e Larva de Stafilinidae no sistema de plantio convencional e de Galumnidae, Parasitidae, Hypogastrura sp., Larva de Stafilinidae e Entomobryidae no sistema de plantio direto pode estar relacionada com a persistência dos residuos dos herbicidas fluazifop-p-butyl e fomesafen nos solos. Estudos demonstraram que o herbicida fluazifop-p-butyl é altamente adsorvido nas partículas coloidais da argila, podendo ser encontrado até a $15 \mathrm{~cm}$ de profundidade no solo, e que sua meia-vida, dependendo da temperatura e da umidade, varia de uma a duas semanas (Gessa et al., 1987; WSSA, 1994; Kulshrestha et al., 1995). Com relação à persistência dos residuos de fomesafen, estudos têm demonstrado que esse produto é moderadamente móvel no solo, sendo rapidamente decomposto em condições anaeróbicas, com meia-vida de três semanas. Já em condições aeróbicas, a meia-vida alcança de 6 a mais de 12 meses, dependendo do tipo de solo (Weber, 1993).
Diante das atuais preocupações globais com a preservação da diversidade biológica e a implementação de estratégias agrícolas ecologicamente sustentáveis, o impacto observado tanto dos sistemas de cultivo quanto dos herbicidas fomesafen + fluazifop sobre as comunidades de artrópodes do solo pode representar importante ferramenta no manejo integrado de pragas, o qual se baseia na adoção de um ou mais métodos de controle que sejam eficientes, de menor custo e interferência no meio ambiente, objetivando reduzir populações de organismos não-benéficos nas lavouras em níveis aceitáveis, observando os critérios econômico, social e ecológico.

\section{LITERATURA CITADA}

ANDERSEN. A. Plant protection in spring cereal production with reduced tillage, II, Pests and beneficial insects. Crop Protec., v. 18, p. 651-657, 1999.

ANDOW, D. A. Vegetational diversity and arthropod population response. Ann. Rev. Entomol, v. 36, p. 561-586, 1991.

ARAÚJO, R. A. et al. Impacto causado por deltametrina em coleópteros de superfície do solo associados à cultura do milho em sistemas de plantio direto e convencional.

Neotrop. Entomol., v. 33, n. 3, p. 379-385, 2004.

BELDEN. J. B.; LYDY, M. J. Impact of atrazine on organophosphate insecticide toxicity. Environ. Toxicol. Chem., v. 19, n. 9, p. 2266-2274, 2000.

CAPINERA, J. L. Relationships between insect pests and weeds: an evolutionary perspective. Weed Sci., v. 53, p. 892-901, 2005.

CIVIDANES, F. J. Efeitos do sistema de plantio e da consorciação soja-milho sobre artrópodes capturados no solo. Pesq. Agropec. Bras., v. 37, n. 1, p. 15-23, 2002.

CLARK, M. S.; GAGE, S. H.; SPENCE, J. R. Habitats and management associated with common ground beetles (Coleoptera: Carabidae) in a Michigan agricultural landscape. Environ. Entomol., v. 26, p. 519-527, 1997.

CONAB. Indicadores da agropecuária. Disponível em: $<$ http://www.conab.gov.br>. Acesso em: 31 mar. 2006.

CRUZ, I. et al. Pragas da cultura do milho em condições de campo: métodos de controle e manuseio de defensivos. Sete Lagoas: Embrapa-CNPMS, 1986. 35 p. (Circular Técnica, 10) 
EMPRESA BRASILEIRA DE PESQUISA AGROPECUÁRIA - EMBRAPA. Centro Nacional de Pesquisa de Solos. Manual de métodos de análise de solo. 2.ed. Rio de Janeiro: 1997. 212 p.

GESSA, C. et al. Interaction of fluazifop-butyl with homoionic clays. Soil Sci., v. 144, p. 420-424, 1987.

GILLER, K. E. et al. Agricultural intensification soil biodiversity and ecosystem function. Appl. Soil Ecol., v. 6, p. 3-16, 1997.

GREEN, R. H. Application of repeated measures designs in environmental impact and monitoring studies. Aust. J. Ecol., v. 18 , p. 81-98, 1993.

KULSHRESTHA, U. C. et al. Wet-only and bulk deposition studies at NewDelhi (India). Water Air Soil Pollut., v. 85, p. 2137-2142, 1995.

MACLAUGHLIN. A.; MINEAU, P. The impact of agricultural practices on biodiversity. Agric. Ecosys. Environ., v. 55, p. 210-212, 1995.

PEREIRA, J. L. et al. Effects of herbicide and insecticide interaction on soil entomofauna under maize crop. J. Environ. Sci. Health, Part B: Pestic., Food Contam., Agric. Wastes, v. B40, n. 1, p. 43-52, 2004.

PHILLIPS, L. D.; COBB, T. P. Effects of habitat structure and lid transparency on pitfall catches. Environ. Entomol., v. 34, n. 4, p. 875-882, 2005.

RAPASSI, R. M. A. et al. Análise econômica comparativa após um ano de cultivo do feijoeiro irrigado, no inverno, em sistemas de plantio convencional e direto, com diferentes fontes e doses de nitrogênio. Bragantia, v. 62, p. 397-404, 2003.

RISCH, S. J.; WRUBEL, R.; ANDOW, D. F. Foraging by a predaceous beetle, Coleomegilla maculata (Coleoptera: Coccinellidae), in a polyculture: effects of plant density and diversity. Environ. Entomol., v. 11, p. 949-950, 1982.

ROBERTSON. L. N.; KETTLE, B. A.; SIMPSON. G. B. The influence of tillage practices on soil macrofauna in a semiarid ecosystem in northeastern Australia. Agric. Ecosys. Environ., v. 48, p. 149-156, 1994.
SALTON, J. C.; MIELNICZUK, J. Relações entre sistemas de preparo, temperatura e umidade de um Podzólico Vermelho-Escuro de Eldorado do Sul (RS). R. Bras. Ci. Solo, v. 19, n. 2, p. 313-319, 1995.

SANDERS, N. J.; GORDON, D. H. The interactive effects of climate, life history, and interspecific neighbours on mortality in a population of seed harvester ants. Ecol. Entomol., v. 29, p. 632-637, 2004.

SAS Institute. SAS user's guide: statistics, version 8.2. 6.ed. Cary: Todd and Browde, 2001.

SILVA, A. A. et al. Controle de plantas daninhas. Brasília: ABEAS, 2003. 260 p. (Módulo 3)

SMITH, H. A.; MCSORLEY, R. Intercropping and pest management: a review of major concepts. Am. Entomol., v. 46, p.154-161, 2000.

SOARES, J. J.; BRAZ, B. A.; BUSOLI, A. C. Impacto de herbicidas sobre artrópodos benéficos associados ao algodoeiro. Pesq. Agropec. Bras., v. 30, n. 9, p. 1135-1140, 1995.

STRONG, D. R.; LAWTON, J. H.; SOUTHWOOD, R. Insects on plants: community patterns and mechanisms. Cambridge: Harvard University Press, 1984. 330 p.

TILL, D. Lipid biosynthesis inhibitors. In: Herbicide action course. West Laffayette: Purdue University, 2003. p. 293-347.

WALKER, C. A. et al. Principles of ecotoxicology. 2.ed. London. Taylor and Francis, 2001. 309 p.

WARDLE, D. A.; YEATES, G. W. The dual importance of competition and predation as regulating forces in terrestrial ecosystems: evidence from decomposer food Webs.

Oecologia, v. 93, p. 303-306, 1993.

WEBER, J. B. Ionization and sorption of Fomesafen and Atrazine by soils and soil Constituents. Pest. Sci., v. 39, p. 31-38, 1993.

WELLER, S. Diquat, paraquat, diphenylethers and oxidiazon - uses and mechanism of action. In: Herbicide action course. West Laffayette: Purdue University, 2003. p. 185-224.

WSSA, 1994. Herbicide handbook Champaign: Weed Society of America. 352 p. 